OPEN ACCESS

Edited by:

Aifu Lin,

Zhejiang University, China

Reviewed by:

Shengtao Zhu,

Capital Medical University, China

Bei Pan,

Nanjing Medical University, China

*Correspondence:

Dong-Qiu Dai daidq63@163.com

Specialty section:

This article was submitted to

Molecular Diagnostics and

Therapeutics,

a section of the journal

Frontiers in Molecular Biosciences

Received: 23 December 2020

Accepted: 26 August 2021

Published: 10 September 2021

Citation:

Zhang C, Li Y-Z and Dai D-Q (2021) Aberrant DNA Methylation-Mediated FOXF2 Dysregulation Is a Prognostic

Risk Factor for Gastric Cancer.

Front. Mol. Biosci. 8:645470.

doi: 10.3389/fmolb.2021.645470

\section{Aberrant DNA Methylation-Mediated FOXF2 Dysregulation Is a Prognostic Risk Factor for Gastric Cancer}

\author{
Cheng Zhang, Yong-Zhi Li and Dong-Qiu Dai * \\ Department of Gastrointestinal Surgery, The Fourth Affiliated Hospital of China Medical University, Shenyang, China
}

Background: The prognosis of gastric cancer (GC) patients is poor. The effect of aberrant DNA methylation on FOXF2 expression and the prognostic role of FOXF2 methylation in GC have not yet been identified.

Methods: The RNA-Seq and gene methylation HM450 profile data were used for analyzing FOXF2 expression in GC and its association with methylation level. Bisulfite sequencing PCR (BSP) was performed to measure the methylation level of the FOXF2 promoter region in GC cell lines and normal GES-1 cells. The cells were treated with the demethylation reagent 5-Aza-dC, and the mRNA and protein expression levels of FOXF2 were then measured by qRT-PCR and western blot assays. The risk score system from SurvivalMeth was calculated by integrating the methylation level of the cg locus and the corresponding Cox regression coefficient.

Results: FOXF2 was significantly downregulated in GC cells and tissues. On the basis of RNA-Seq and Illumina methylation 450 data, FOXF2 expression was significantly negatively correlated with the FOXF2 methylation level (Pearson's $R=-0.42, p<$ $2.2 \mathrm{e}^{-16}$ ). The FOXF2 methylation level in the high FOXF2 expression group was lower than that in the low FOXF2 expression group. The BSP assay indicated that the methylation level of the FOXF2 promoter region in GC cell lines was higher than that in GES-1 cells. The qRT-PCR and western blot assay showed that FOXF2 mRNA and protein levels were increased in GC cells following treatment with 5-Aza-Dc. The methylation risk score model indicated that patients in the high risk group had poorer survival probability than those in the low risk group ( $\mathrm{HR}=1.84(1.11-3.07)$ and $p=0.0068)$. FOXF2 also had a close transcriptional regulation network with four miRNAs and their corresponding target genes. Functional enrichment analysis of the target genes revealed that these genes were significantly related to several important signaling pathways.

Conclusion: FOXF2 was downregulated due to aberrant DNA methylation in GC, and the degree of methylation in the promoter region of FOXF2 was related to the prognosis of patients. The FOXF2/miRNAs/target genes axis may play a vital biological regulation role in GC. 


\section{INTRODUCTION}

Gastric cancer (GC) remains an important cancer worldwide, with a large number of new cases and deaths each year, making it the fifth most frequently diagnosed cancer and the third leading cause of cancer-related deaths (Bray et al., 2018). As the main risk factor for GC, approximately $90 \%$ of new GC cases are attributed to Helicobacter pylori infection (Plummer et al., 2015). Other risk factors include high salt diet, low-fruit diet, drinking, and smoking (IARC, 2012). Although the morbidity and mortality of GC are slowly decreasing due to advances in the diagnosis and treatment of GC in recent years (Zhang et al., 2017), the 5-years survival rate of patients with GC is still below $29 \%$ because of the high invasiveness and recurrence of GC (Allemani et al., 2015). Therefore, the study of molecular mechanisms underlying the development and progression of GC is particularly important to establish novel approaches for treating GC.

Epigenetic dysregulation plays an indispensable role in the development and progression of GC (Ebrahimi et al., 2020). In addition to changes and mutations in the genomic DNA sequence, epigenetic changes are observed in the mechanisms of gene expression regulation, including DNA methylation, chromatin remodeling, alteration in noncoding RNA expression, and histone post-translational modifications, and these changes are reversible (Puneet et al., 2018). Aberrant methylation of the $\mathrm{CpG}$ island in the promoter region of the gene plays an important role in its inactivation. Abnormal methylation of the $\mathrm{CpG}$ island in the promoter region of the tumor suppressor gene leads to its transcriptional inhibition, downregulation, or deletion of expression, resulting in the development of GC. Thus, understanding the mechanism of epigenetic changes is essential for the diagnosis, treatment, and prevention of GC (Sonohara et al., 2017).

Forkhead box F2 (FOXF2) is a member of the Forkhead box family of transcription factors, and all FOX proteins can bind to DNA. These transcription factors (TFs) act as an activator or inhibitor of gene transcription with a highly conserved 110amino-acid DNA-binding domain (Coffer and Burgering, 2004; Katoh and Katoh, 2004). FOXF2 is closely related to the regulation of human growth and development and tumors (Aitola et al., 2000; Coffer and Burgering, 2004). Several studies have shown that FOXF2 is a potential tumor suppressor, and it can inhibit epithelial-mesenchymal transition (EMT) and metastasis in breast cancer (Wang et al., 2015). However, the comprehensive upstream and downstream transcriptional regulation roles of FOXF2 in GC remain unclear.

\section{MATERIALS AND METHODS}

\section{RNA Sequencing Data Collection and Processing}

The Cancer Genome Atlas (TCGA, https://portal.gdc.cancer.gov/ ), Genotype-Tissue Expression (GTEx, https://www.gtexportal. org/home/), and The Human Protein Atlas (HPA, https://www. proteinatlas.org/) were used to assess the FOXF2 expression level in various tumor tissues, including GC, and multiple human normal tissues. Expression profiling by array from Gene Expression Omnibus (GEO) was also used to confirm FOXF2 expression in GC. The RNA-Seq data of GC generated from the TCGA database were downloaded using the TCGAbiolinks package (Colaprico et al., 2016) in $\mathrm{R}$ and used to identify differentially expressed genes (DEGs) in GC and normal gastric tissues. The cutoff criteria were fold change $|\mathrm{FC}|>2.0$ and FDR $<0.05$. The Trimmed Mean of $M$ values (TMM) method (Robinson and Oshlack, 2010) was used to normalize the observed counts, and the limma package (Ritchie et al., 2015) was then used for identification with $\mathrm{R}$ software.

\section{GC Cell Lines and Clinical Samples}

Human GC cell lines SGC-7901, BGC-823, MGC-803, and MKN-45 and the normal gastric epithelium cell line (GES-1) used in the present study were purchased from the Chinese Academy of Sciences (Shanghai, China). All cell lines were maintained in DMEM medium (Gibco, Germany) containing $10 \%$ fetal bovine serum (FBS, MilliporeSigma, Shanghai, China), $100 \mu \mathrm{g} / \mathrm{ml}$ streptomycin (Thermo Fisher Scientific, United States), and $100 \mathrm{U} / \mathrm{mL}$ penicillin (Thermo Fisher Scientific) in an incubator with $5 \% \mathrm{CO}_{2}$ at $37^{\circ} \mathrm{C}$. Medium changes were performed every 2-3 days (after reaching $70-80 \%$ cell confluence).

Clinical samples including 25 paired gastric tumor tissues and adjacent nontumor tissues (at least $5 \mathrm{~cm}$ away from the clear edge of tumor tissues) were collected from patients with GC during surgery in the Fourth Affiliated Hospital of China Medical University. None of the patients received any adjuvant treatments before surgery. The ethical approval for this study was obtained from the Fourth Affiliated Hospital of China Medical University, and written informed consents were signed by all patients. All the tissues were removed during the surgery and immediately stored at $-80^{\circ} \mathrm{C}$.

\section{RNA Extraction and Real-Time PCR}

Total RNA was extracted from cells and tissues by using TRIzol reagent (Invitrogen, United States), and the concentration and purity of RNA were measured with a spectrophotometer. Total RNA was converted into cDNA by reverse transcription using the PrimeScript $^{\mathrm{TM}}$ RT Reagent Kit with gDNA Eraser (TaKaRa, Dalian, China). Quantitative real-time PCR (qRT-PCR) was performed using TB Green ${ }^{\circledR}$ Premix Ex Taq ${ }^{\mathrm{TM}}$ II (TaKaRa, Dalian, China). Glyceraldehyde-3-phosphate dehydrogenase (GAPDH) was used as a reference. The primers used for qRTPCR are shown in Supplementary Table S1. All steps were performed in accordance with the instructions of the reagent manufacturer.

\section{Bisulfite Sequencing PCR (BSP) and Methylation Drug Treatment}

Methprimer 2.0 (Li and Dahiya, 2002) was used to determine whether there are $\mathrm{CpG}$ islands in the promoter sequence of 
FOXF2 and for designing BSP primers. The MiniBEST Universal Genomic DNA Extraction Kit (TaKaRa, Dalian, China) was used to extract DNA from cells and tissues. The EZ DNA MethylationGold kit (Zymo Research, Irvine, CA, United States) was used for methylation to modify DNA. The bisulfite-modified DNA was used for BSP. The primers sequences are shown in Supplementary Table S1. Three GC cell lines, namely SGC7901, MGC-803, and MKN-45, and GES-1 were cultured and treated with 5-Aza-dC (Sigma-Aldrich) at the dose of $15 \mu \mathrm{mol} / \mathrm{L}$ for 3 days. The mRNA and protein expression levels of FOXF2 were measured.

\section{Cytoplasm and Nucleus Separation}

The Nuclear and Cytoplasmic Protein Extraction Kit (Wanleibio, China) was used to isolate cytoplasm and nucleus protein according to the manufacturer's instructions.

\section{Western Blotting}

The protocols for western blotting were described in a previous study (Zhang et al., 2019). The primary antibodies were incubated at $4^{\circ} \mathrm{C}$ overnight according to the following dilution ratio: FOXF2 (1:200; Abcam, Cambridge, MA, United States). $\beta$-actin and Histone $\mathrm{H} 3$ were used as endogenous loading control.

\section{FOXF2 Methylation and Prognosis Risk Analysis}

The Stomach Adenocarcinoma methylation (HM450) profile, downloaded from cBioPortal (Cerami et al., 2012) (http://www. cbioportal.org/), was used to assess correlation between FOXF2 expression and methylation level. The cg locus located at the FOXF2 promoter region was identified using the MEXPRESS tool (Koch et al., 2019), and the association between the methylation level of the target cg locus and FOXF2 expression was then evaluated. SurvivalMeth (Zhang et al., 2020) (http://biobigdata.hrbmu.edu.cn/survivalmeth), a web server to investigate the effect of DNA methylation-related functional elements on prognosis, was used to analyze methylation levels and the correlation between methylation and risk score, gene expression, and prognosis. All steps were performed in accordance with the developer's instructions. The risk score system from SurvivalMeth was calculated by integrating the methylation level of the cg locus and the corresponding Cox regression coefficient. The optimal cutoff value of risk score was then identified by the "Maximally Selected Rank Statistics" (maxstat) model (Laska et al., 2012). Then, the patients were divided into low/high risk groups based on the risk score, and the survival analysis was performed to determine the prognosis of the two groups.

\section{FOXF2-miRNAs and Target Genes Prediction Network}

The inclusion criteria for FOXF2-miRNAs-target gene regulation network are as follows: 1) TransmiR v2.0 database (Tong et al., 2019) (http://www.cuilab.cn/transmir) is a database that provides information on the regulatory relationships between TFs and miRNAs. Thus, we first obtained the miRNAs that could be transcriptionally regulated by FOXF2; 2) According to the cutoff criteria of FC $>2.0$ and FDR $<0.05$, the upregulated miRNAs in GC were identified by analyzing the TCGA Stomach Adenocarcinoma mature miRNA expression profile; 3) Pearson's correlation coefficient ( $\mathrm{R}$ value) for association between FOXF2 and miRNAs is less than -0.3 ; and 4) Upregulated miRNAs (FC $>2.0$ and FDR $<0.05$ ) in blood of patients with GC determined by analyzing the blood miRNA expression profile from the BBCancer database (Zuo et al., 2020). The miRNAs that met the four abovementioned criteria were considered to be FOXF2 transcriptional regulated miRNAs. Then, Starbase v2.0 (Li et al., 2014) (http://starbase.sysu.edu.cn/) was used to predict the target genes of miRNAs. The predicted target genes that are downregulated in GC were included for regulation network construction.

\section{Gene Function Enrichment Analysis}

To determine the biological function of these target genes, we performed biological pathway analysis by FunRich, which is a functional enrichment and interaction network analysis tool (Pathan et al., 2015). The pathway items with $p<0.05$ were considered to be statistically significant.

\section{Statistical Analyses}

All statistical analyses were performed with SPSS version 19.0 (Chicago, IL, United States) or GraphPad Prism software version 7 (GraphPad Software Inc., CA, United States). The statistical significance between two groups was analyzed by paired or unpaired $t$ test. The Kaplan-Meier curve was analyzed by the log-rank test. Receiver operating characteristic (ROC) curves were used to test the diagnostic ability of blood miRNAs in GC. $p<0.05$ was considered to be statistically significant.

\section{RESULTS}

\section{FOXF2 is Downregulated in GC Cells and Tissues}

According to the identification of DEGs, there were 2,878 highly expressed genes and 2,710 low expressed genes in GC (Figure 1A). We then analyzed 373 cases of GC tissues and 32 cases of normal gastric tissues from the TCGA database. Compared to normal tissues, the expression of FOXF2 in GC tissues was significantly decreased (Figure 1B). Three expression profiling data from GEO datasets also showed that FOXF2 was down-regulated in GC tissues (Figure 1C). The subcellular locations prediction from COMPARTMENTS (Binder et al., 2014) showed that FOXF2 was mainly located in the nucleus, cytoplasm and extracellular. (Figure 1D). Cytoplasm and nucleus protein separation assay found that FOXF2 protein was both detected in cytoplasm and nucleus (Figure 1E). Moreover, by performing qPCR on four types of GC cells, namely MKN-45, SGC-7901, MGC-803, BGC-823, and the 

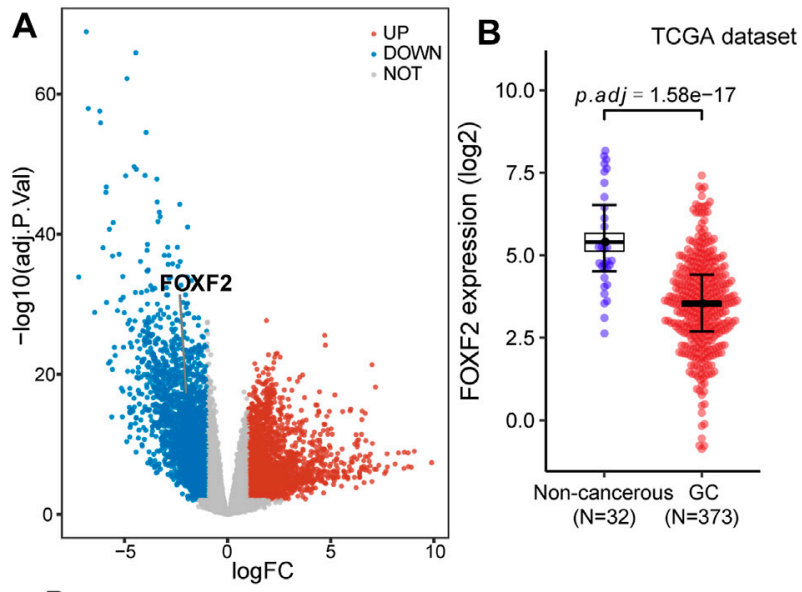

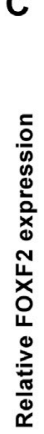
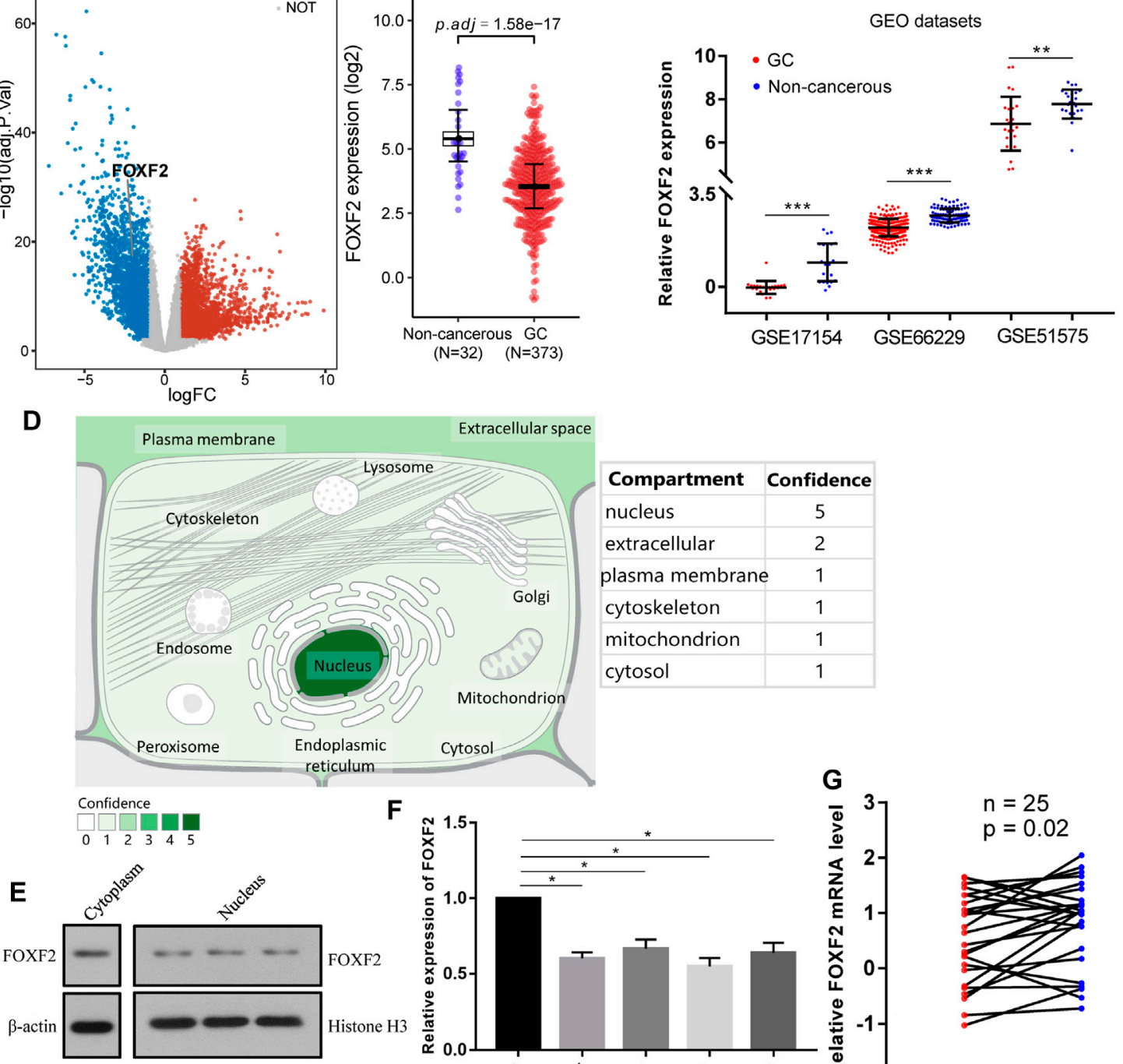

\section{Compartment Confidence}

nucleus

extracellular

plasma membrane

cytoskeleton

mitochondrion

cytosol

Mitochondrion

Cytosol

F
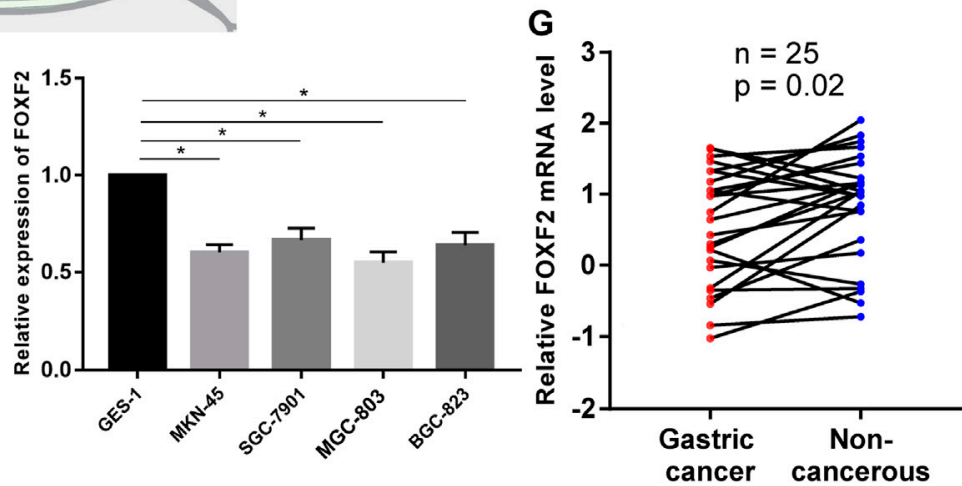

FIGURE 1 | FOXF2 was downregulated in GC cells and tissues. (A) A total of 5,588 differentially expressed genes (2,878 highly and 2,710 low expressed genes) were identified by analyzing GC RNA-Seq data from the TCGA dataset. (B) According to the TCGA dataset, FOXF2 was significantly downregulated in $373 \mathrm{GC}$ tissues. (C) Three expression profiling data from GEO datasets showed that FOXF2 was down-regulated in GC tissues. (D) The subcellular location prediction from COMPARTMENTS showed that FOXF2 has the greatest possibility of mainly being located in the nucleus, cytoplasm and extracellular. (E) The cytoplasm and nucleus protein separation assay found that FOXF2 protein was both detected in cytoplasm and nucleus. (F,G) The qRT-PCR assay indicated that FOXF2 was significantly downregulated in GC cells and tissues. ${ }^{*} p<0.05,{ }^{* *} p<0.01$, and ${ }^{\star \star *} p<0.001$.

normal gastric tissue cell line GES-1 and 25 pairs of GC tissues and adjacent normal tissues, we determined that the expression of FOXF2 is significantly downregulated in GC cells and tissues (Figures 1F,G). By using the HPA, TCGA, and GTEx databases, we analyzed the expression level of FOXF2 in various human tumor tissues and normal tissues. The results indicated that FOXF2 had quite lower expression abundance in tumor tissues and its expression varied in different types of tissues (Figures 2A-C).

\section{FOXF2 Dysregulation is Accompanied by Aberrant DNA Methylation}

By analyzing TCGA RNA-Seq and Illumina methylation HM450 data, there are a total of 372 GC samples with 


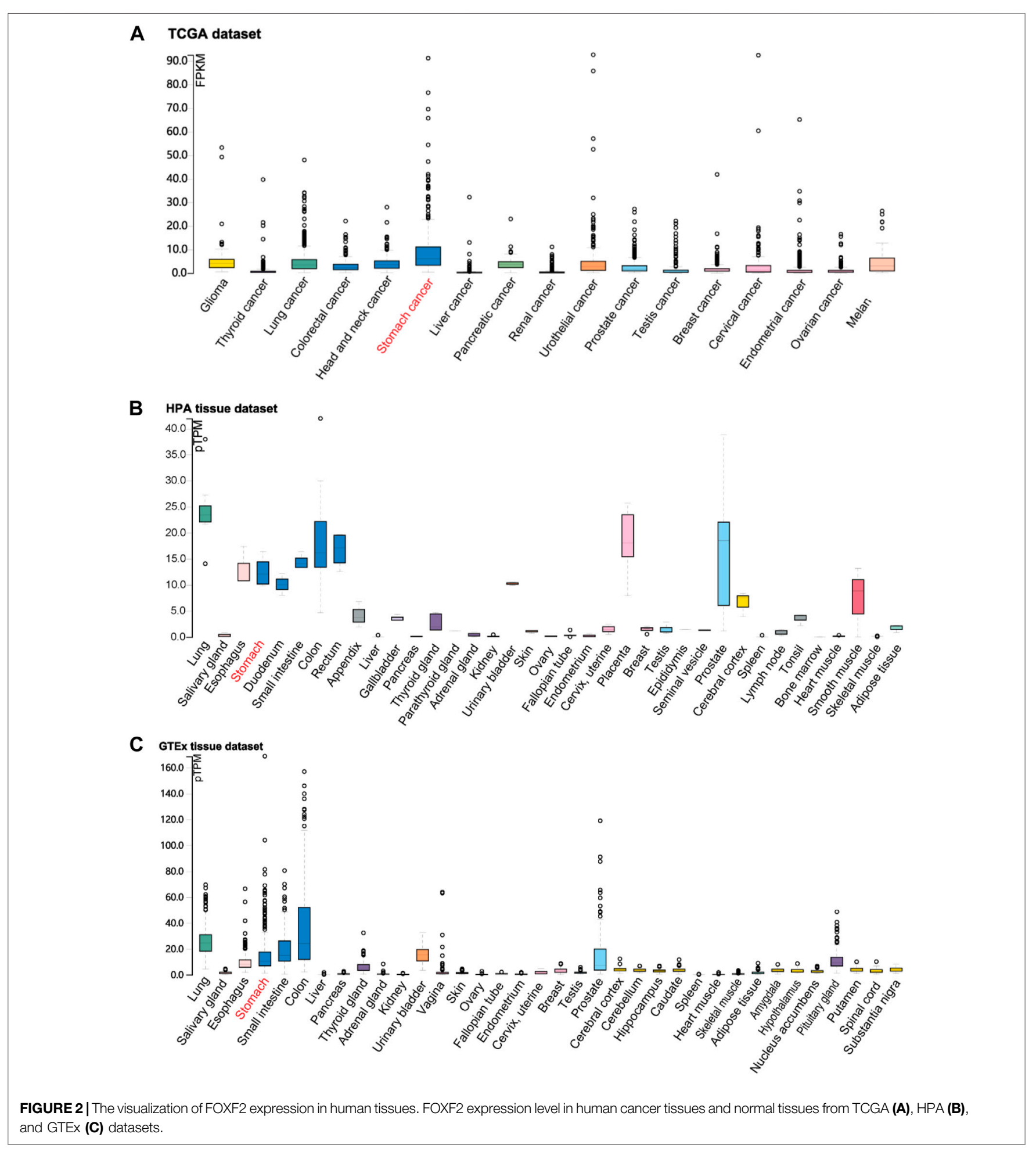

valid FOXF2 expression and methylation beta data. Pearson analysis found that FOXF2 expression was significant negatively correlated with the FOXF2 methylation level (Figure 3A, Pearson $\mathrm{R}=-0.42, p<2.2 \mathrm{e}-16$ ). According to the median value of FOXF2 expression, 372 GC samples were divided into low or high FOXF2 expression group. The unpaired $t$-test indicated that the FOXF2 methylation level in the high FOXF2 expression group was lower than that in the low FOXF2 expression group (Figure 3B). We then divided the 372 GC samples into low (0-1/3), median (1/ 

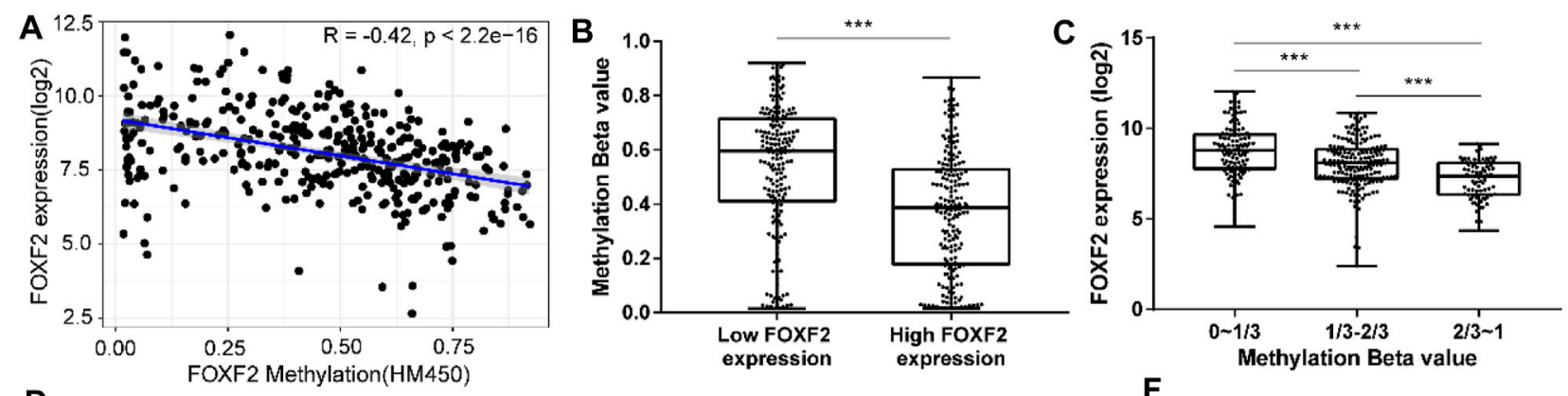

D 190

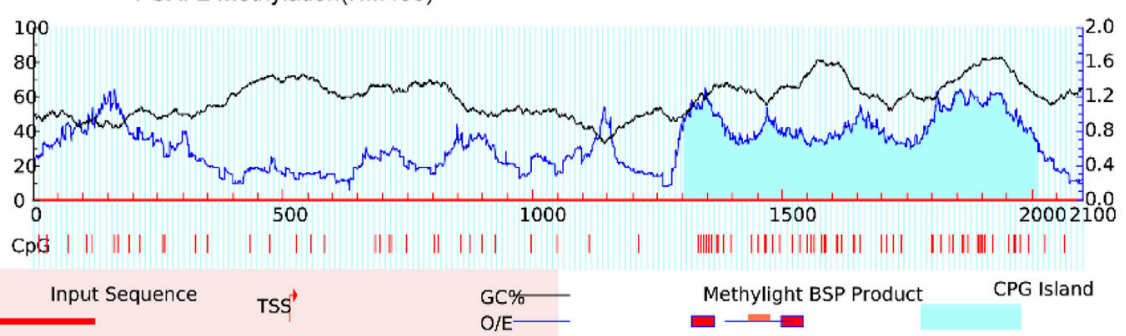

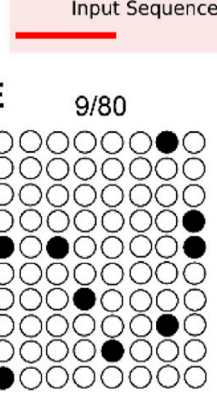

GES-1

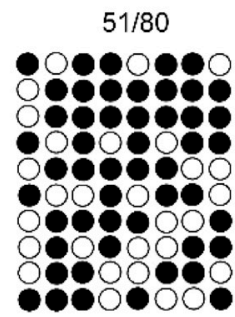

MKN-45

Unmethylate

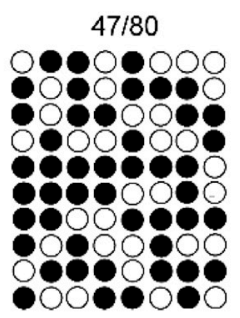

SGC-7901

SGC-7901 $\longrightarrow$
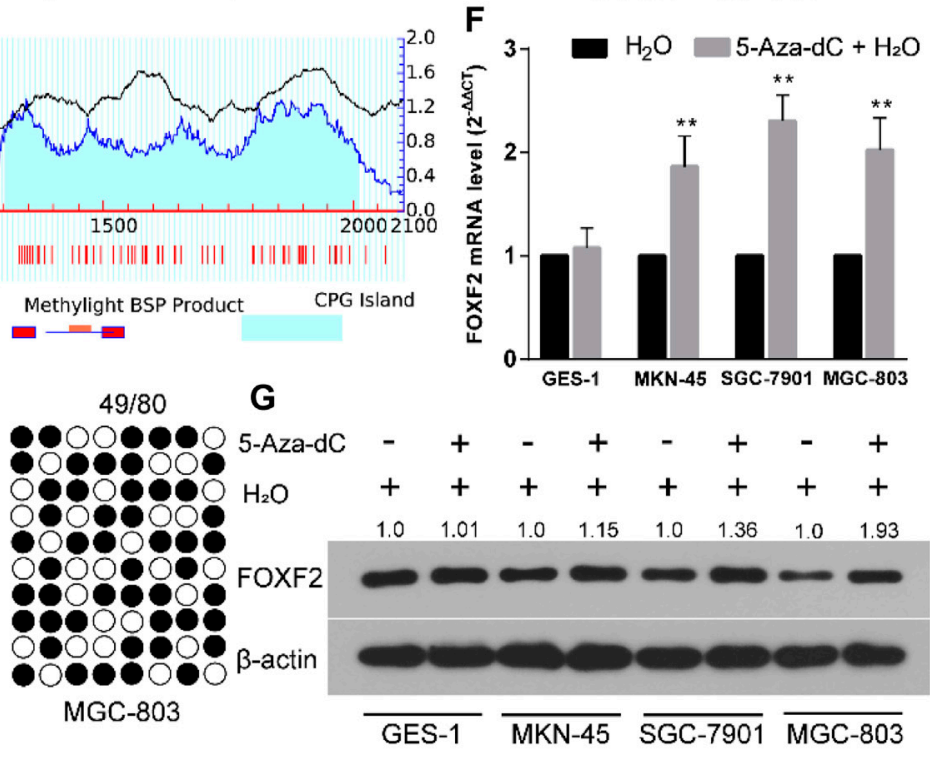

FIGURE 3 | FOXF2 was downregulated through aberrant DNA methylation in the promoter region. (A) FOXF2 expression was negatively correlated with FOXF2 methylation level (Pearson $\mathrm{R}=-0.42, p<2.2 \mathrm{e}^{-16}$ ). (B) A total of $372 \mathrm{GC}$ samples were divided into low or high FOXF2 expression group according to the median value of FOXF2 expression. The unpaired $t$-test suggested that the FOXF2 methylation level in the high FOXF2 expression group was lower than that in the low FOXF2 expression group. (C) The $372 \mathrm{GC}$ samples were divided into low (0-1/3), median (1/3-2/3), and high (2/3-1) methylation groups according to the tertile of the methylation level. The results showed that FOXF2 expression was lowest in the high methylation group and the highest in the low methylation group. (D) A CpG island with the length of $708 \mathrm{bp}$ was found in the promoter region of FOXF2 by Methprimer 2.0 prediction. (E) The BSP assay suggested that the methylation level of the FOXF2 promoter region in GC cell lines (MKN-45, SGC-7901, and MGC-803) was higher than that in the normal control GES-1 cell line. (F and G) qRT-PCR and western blot assay showed that the mRNA and protein levels of FOXF2 were increased in GC cells following treatment with 5-Aza-Dc; however, no difference was found in GES-1 cells. ${ }^{*} p<0.05,{ }^{\star *} p<0.01$, and ${ }^{* \star *} p<0.001$

$3-2 / 3)$, and high (2/3-1) methylation groups according to the tertile of the methylation level. The results showed that FOXF2 expression was the lowest in the high methylation group and the highest in the low methylation group (Figure 3C). Thus, we speculated that FOXF2 may be misregulated by aberrant DNA methylation.

The sequence from upstream $-2,000$ to downstream $+100 \mathrm{bp}$ relative to the transcription start site (TSS) of FOXF2 was regarded as the promoter region. Methprimer 2.0 predicted a CpG island (708 bp) in the promoter region of FOXF2 (Figure 3D). The methylation level of the FOXF2 promoter region in GES-1 and 3 GC cell lines was measured by the BSP assay. The results suggested that the methylation level of the FOXF2 promoter region in GC cell lines (MKN-45, SGC-7901, and MGC-803) was higher than that in the normal control GES-1 cell line (Figure 3E). These cells were then treated with the demethylation reagent $5-\mathrm{Aza}-\mathrm{dC}$ to assess the effect of methylation level on FOXF2 expression. qRT-PCR and western blot assay showed that the mRNA and protein levels of FOXF2 were increased in GC cells following treatment with 5Aza-Dc; however, the difference was not found in GES-1 cells (Figures 3F,G).

\section{FOXF2 is a Prognostic index for Patients With GC}

To further explore the methylation status of the FOXF2 cg locus, we analyzed the association between the methylation level of the target cg locus and FOXF2 expression using the MEXPRESS tool. It was found that the methylation levels of most of the cg loci in the low FOXF2 expression group were significantly higher than those in the high FOXF2 expression group (Figure 4A). A total of $6 \mathrm{cg}$ loci located at the FOXF2 promoter region were identified by the SurvivalMeth web 

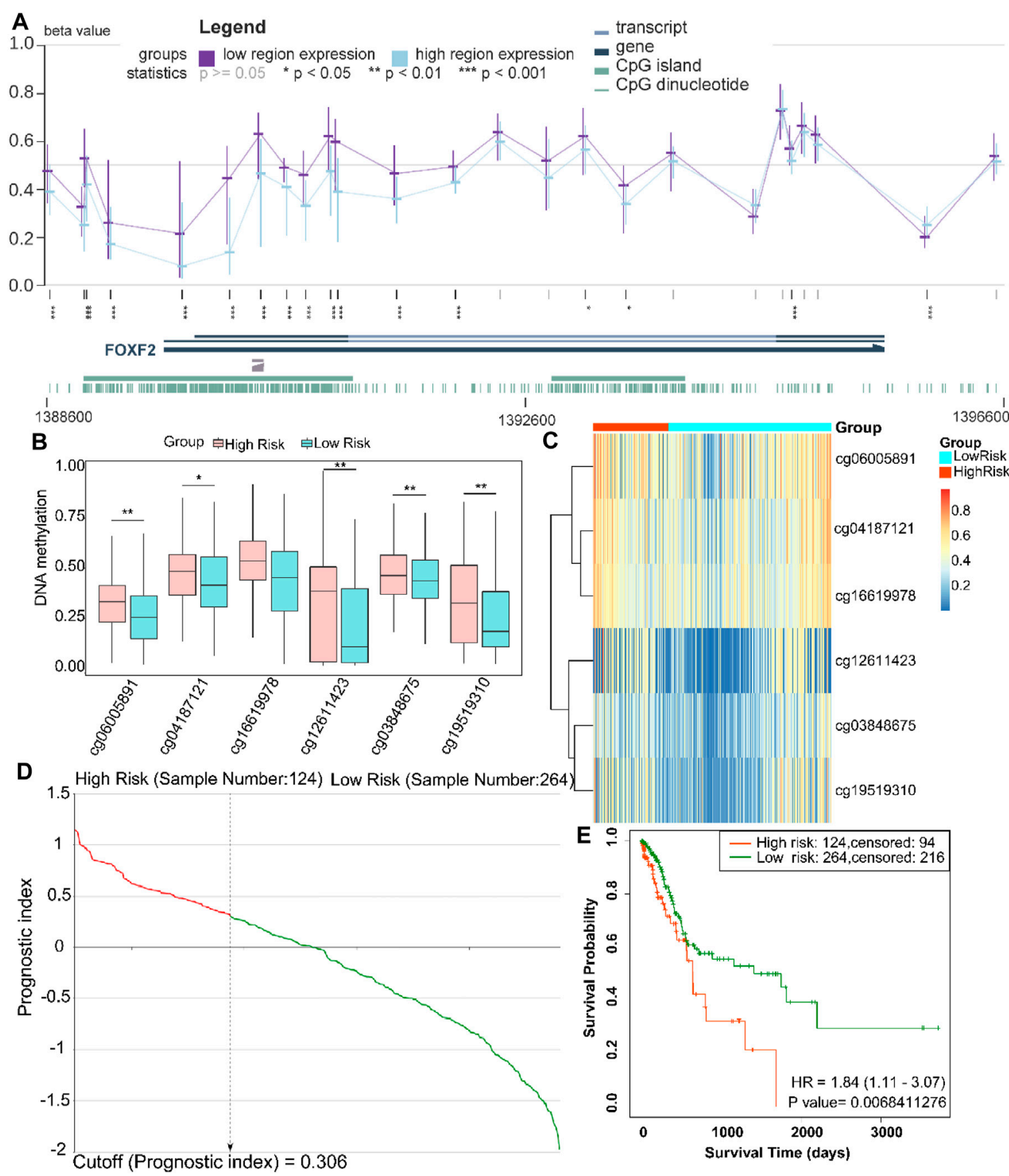

FIGURE 4 |FOXF2 is a prognostic index for patients with GC patients. (A) The methylation levels of most of the cg loci in the low FOXF2 expression group were significantly higher than those in the high FOXF2 expression group as determined from MEXPRESS. (B,C) SurvivalMeth data showed that the methylation levels of 5 promoter region cg loci (cg06005891, cg04187121, cg12611423, cg03848675, and cg19519310) in the high risk group were significantly higher than those in the low risk group. The methylation level of cg16619978 in the high risk group was also higher than that in the low risk group; however, the difference was not significant. (D) At the optimal cutoff prognostic index of 0.306, a total of $388 \mathrm{GC}$ samples were divided into high or low risk group. (E) The Kaplan-Meier analysis showed that patients in the high risk group had a poorer survival probability than those in the low risk group (HR $=1.84(1.11-3.07)$ and $p=0.0068) .{ }^{*} p<0.05,{ }^{* *} p<0.01$, and ${ }^{* \star} p<0.001$.

server. The detailed information of the cg locus is shown in Table 1. SurvivalMeth showed that the methylation levels of 5 cg loci (cg06005891, cg04187121, cg12611423, cg03848675, and $\mathrm{cg} 19519310)$ in the high risk group were significantly higher than those in the low risk group (Figures $4 \mathrm{~B}, \mathrm{C}$ ). Although the difference for cg16619978 was found to be nonsignificant, its methylation level in the high risk group was also higher than that in the low risk group. According to 
TABLE 1 | Information on the $6 \mathrm{cg}$ loci in the FOXF2 promoter region.

\begin{tabular}{lcccl}
\hline Cg locus & Chromosome & Location & Gene location & Island \\
\hline cg03848675 & 6 & 1389146 & TSS1500 & Island \\
cg04187121 & 6 & 1388858 & TSS1500 & N shore \\
cg06005891 & 6 & 1389167 & TSS1500 & Island \\
cg12611423 & 6 & 1389966 & TSS200 & Island \\
cg16619978 & 6 & 1388806 & TSS1500 & N shore \\
cg19519310 & 6 & 1389367 & TSS1500 & Island
\end{tabular}

SurvivalMeth, a total of 388 GC samples were divided into a high or low risk group at the optimal cutoff prognostic index (Figure 4D). The Kaplan-Meier analysis showed that patients in the high risk group had a poorer survival probability than those in the low risk group (Figure 4E, HR $=1.84(1.11-3.07$ ) and $p=0.0068$ ). All these findings indicated that FOXF2 can be a promising prognostic index in GC.

\section{FOXF2-miRNAs-Target Genes Regulation Network Construction}

According to the previous inclusion criteria, we obtained four potential target miRNAs, including miR-17-5p, miR-182-5p, miR183-5p, and miR-503-5p (Figure 5A). We found that compared to normal people without cancer, patients with GC showed significant upregulation of these four miRNAs in their blood (Figure 5B), which aroused our interest. ROC analysis showed that these four miRNAs had preferable ability in distinguishing patients with GC from normal individuals. The area under the curve (AUC) values were greater than 0.7 , which indicated that the four miRNAs could be used as biomarkers for the diagnosis of GC (Figure 5C). Pearson's correlation analysis showed that FOXF2 expression was significantly negatively correlated with miR-17-5p, miR182-5p, miR-183-5p, and miR-503-5 expression (Figure 5D). Next, FOXF2-miRNAs-target genes regulation network was constructed, and it showed that FOXF2 had a close regulation relationship with the miRNAs and the corresponding target genes (Figure 5E). We then performed functional enrichment analysis of these 95 genes and found that these genes are significantly related to many important signaling pathways, including HIF-1a, ErbB, mTOR, EMT, Akt, VEGF, and VEGFR-mediated signaling pathway (Figure 5F).

\section{DISCUSSION}

It is known that epigenetics plays an indispensable role in the complex molecular mechanism of GC occurrence and development (Chia and Tan, 2016; Huang et al., 2018). Our team has conducted many studies on this topic (Zhang et al., 2019; Liang et al., 2020). In the present study, by exploring public databases, we found that FOXF2 was expressed to varying degrees in a variety of normal and cancer tissues in the human body. Compared to the normal gastric tissue, FOXF2 expression was significantly downregulated in GC tissues. We confirmed this through qRT-PCR assay at the cell and tissue level. In addition, we found that the downregulation of FOXF2 in GC may be due to abnormal methylation of the promoter region of FOXF2, and the methylation of the FOXF2 promoter was related to the prognosis of patients.

Many studies have shown that FOXF2 was dysregulated in different types of cancers and can be regulated by aberrant DNA methylation (Tian et al., 2015; Chen et al., 2017). FOXF2 was shown to be downregulated in GC cell lines and tissues. However, its dysregulation mechanism in GC has not yet been fully elucidated. First, we found that FOXF2 expression was significantly negatively correlated with the FOXF2 methylation level. There was also a $\mathrm{CpG}$ island in the promoter region of FOXF2. Thus, we speculated that FOXF2 may be regulated by aberrant DNA methylation. The BSP assay suggested that the methylation level of the FOXF2 promoter region in GC cell lines was higher than that in the normal GES-1 cell line. The cells were then treated with 5-Aza-Dc to investigate the expression change of FOXF2 induced by methylation. qRT-PCR and western blot assay revealed that the mRNA and protein levels of FOXF2 were increased in GC cells following treatment with 5-Aza-Dc. The demethylated drug 5-Aza-Dc could decrease the aberrant DNA methylation status in the gene promoter region, thus improving FOXF2 expression.

FOXF2, as a transcriptional regulator, was also found to have a transcriptional repression effect on the miR-200b 200a $\sim 429$ locus in lung cancer (Kundu et al., 2016). Lu et al. (2020) also reported that FOXF2 can negatively regulate the transcription of TGF- $\beta$ isoforms (TGFB1, TGFB2, and TGFB3) in basal-like breast cancer. Thus, FOXF2 may play a tumor suppressor role through transrepressing gene expression. By integrating the miRNA expression profile and TF-miRNA regulation from the TransmiR database, four miRNAs (miR-17-5p, miR-182-5p, miR-183-5p, and miR-503-5p) were identified to be the transcriptional regulation targets of FOXF2. These four miRNAs were also significantly upregulated in the blood of patients with GC as compared to that in normal people. The blood miRNA expression profile from the BBCancer database contains 115 GC and 2,759 non-cancer samples, which makes the ROC findings more reliable. Accumulating evidences revealed that the four miRNAs can play vital roles in GC tumorigenesis and development (Lin et al., 2019; Yuan et al., 2019; Huang et al., 2020; Song et al., 2020; Zhang et al., 2020). However, the expression condition in GC patient blood and dysregulated mechanism in transcriptional regulation are still not completely understood. Our findings provide evidence for studying the transcription regulation of theses miRNAs by FOXF2. As expected, these four target genes could be used as biological indicators for clinical diagnosis.

The dysregulation of FOXF2 also plays an indispensable role in tumorigenesis and development of other types of cancer. Hauptman et al. found that FOXF2 was highly methylated and downregulated in colorectal cancer, which can be used as a biomarker for the diagnosis of colorectal cancer (Hauptman et al., 2019). Zhang and Chen et al. found that miR-182 and miR-130a were highly expressed in colorectal cancer and can decrease the target FOXF2 expression, thereby promoting the proliferation, invasion, and metastasis of colorectal cancer 


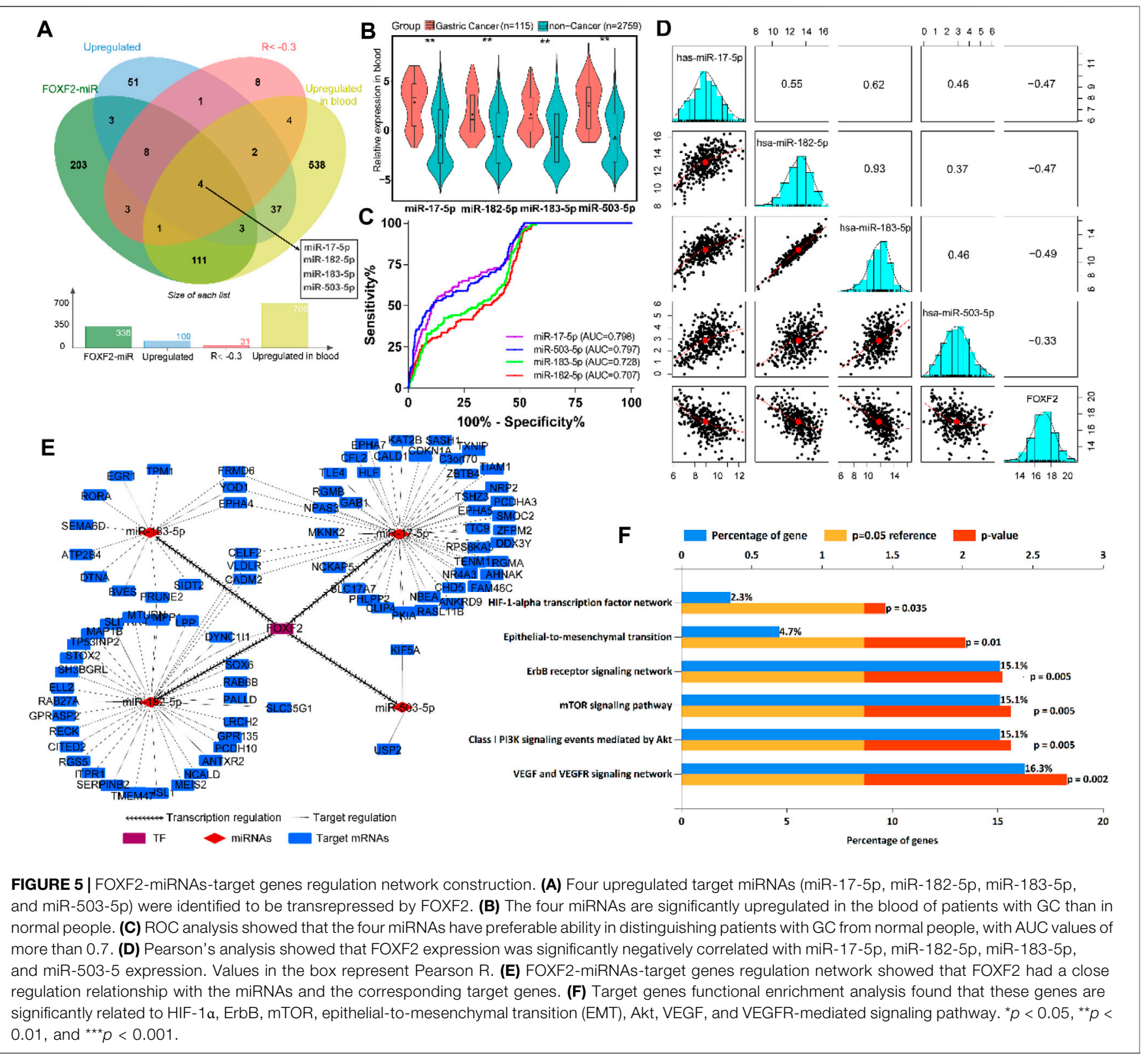

(Zhang et al., 2015; Chen et al., 2017). Kong et al. (2016) found that FOXF2 was downregulated in non-small cell lung cancer, and the expression of FOXF2 was related to the prognosis of patients. Lo et al. (2016) found that FOXF2 may promote or suppress breast cancer tumorigenesis according to different tumor subtypes. Dou et al. (2017) showed that the downregulation of FOFX2 expression in liver cancer cells increased E-cadherin, decreased vimentin, and induced mesenchymal-epithelial transformation of liver cancer cells, thereby inhibiting their invasion and migration, but it promoted the proliferation of liver cancer cells. Hirata et al. (2013) reported that the expression of FOXF2 increased after knockdown of miR-182-5p, which can inhibit the proliferation, invasion, and metastasis of prostate cancer. In the present study, we found that FOXF2 was downregulated due to aberrant DNA methylation in GC, and the prognosis of patients with GC was related to the degree of methylation in the FOXF2 promoter region.

Our present study had some limitations. Although we verified that FOXF2 was highly correlated with four miRNAs, further experiments to verify the targeting relationship between FOXF2 and the four miRNAs were lacking. In addition, as a biological indicator of GC diagnosis, the four miRNAs need to be further verified clinically. The signaling pathways and functions of FOXF2 in GC also require further experiments to verify. The present study provides a clear direction for our future studies and could assist research on the role of FOXF2 in GC. 
In conclusion, we found that FOXF2 was downregulated due to aberrant DNA methylation in GC and that the degree of methylation in the promoter region of FOXF2 was related to the prognosis of patients. Moreover, the four miRNAs, which could be transrepressed by FOXF2, may be used as biological indicators for the diagnosis of GC. These findings showed that FOXF2 can be a useful target in revealing the tumorigenesis process of GC. The methylation of FOXF2 can also serve as a prognostic risk factor for GC.

\section{DATA AVAILABILITY STATEMENT}

Publicly available datasets were analyzed in this study. This data can be found here: RNA-Seq and DNA methylation HM450 data were downloaded from the TCGA (https://portal.gdc.cancer.gov/), GTEx (https://www.gtexportal.org/home/), HPA (https://www. proteinatlas.org/) database, Gene Expression Omnibus (accession numbers: GSE17154, GSE66229 and GSE51575) and cBioPortal database (http://www.cbioportal.org/), respectively.

\section{ETHICS STATEMENT}

The studies involving human participants were reviewed and approved by the Fourth Affiliated Hospital of China Medical University. The patients/participants provided their written informed consent to participate in this study.

\section{REFERENCES}

Aitola, M., Carlsson, P., Mahlapuu, M., Enerback, S., and Pelto-Huikko, M. (2000). Forkhead transcription factorFoxF2 is expressed in mesodermal tissues involved in epithelio-mesenchymal interactions. Dev. Dyn. 218 (1), 136-149. doi:10.1002/(sici)1097-0177(200005)218:1<136::aid-dvdy12>3.0.co;2-u

Allemani, C., Weir, H. K., Carreira, H., Harewood, R., Spika, D., Wang, X.-S., et al. (2015). Global surveillance of cancer survival 1995-2009: analysis of individual data for 25676887 patients from 279 population-based registries in 67 countries (CONCORD-2). The Lancet 385 (9972), 977-1010. doi:10.1016/ S0140-6736(14)62038-9

Binder, J. X., Pletscher-Frankild, S., Tsafou, K., Stolte, C., O’Donoghue, S. I., Schneider, R., et al. (2014). COMPARTMENTS: unification and visualization of protein subcellular localization evidence. Database 2014, bau012. doi:10.1093/ database/bau012

Bray, F., Ferlay, J., Soerjomataram, I., Siegel, R. L., Torre, L. A., and Jemal, A. (2018). Global cancer statistics 2018: GLOBOCAN estimates of incidence and mortality worldwide for 36 cancers in 185 countries. CA: A Cancer J. Clinicians 68 (6), 394-424. doi:10.3322/caac.21492

Cerami, E., Gao, J., Dogrusoz, U., Gross, B. E., Sumer, S. O., Aksoy, B. A., et al. (2012). The cBio Cancer Genomics Portal: An Open Platform for Exploring Multidimensional Cancer Genomics Data: Figure 1. Cancer Discov. 2 (5), 401-404. doi:10.1158/2159-8290.CD-12-0095

Chen, W., Tong, K., and Yu, J. (2017). MicroRNA-130a is upregulated in colorectal cancer and promotes cell growth and motility by directly targeting forkhead box F2. Mol. Med. Rep. 16 (4), 5241-5248. doi:10.3892/mmr.2017.7257

Chen, X., Hu, H., Liu, J., Yang, Y., Liu, G., Ying, X., et al. (2017). FOXF2 promoter methylation is associated with prognosis in esophageal squamous cell carcinoma. Tumour Biol. 39 (2), 101042831769223. doi:10.1177/ 1010428317692230

Chia, N.-Y., and Tan, P. (2016). Molecular classification of gastric cancer. Ann. Oncol. 27 (5), 763-769. doi:10.1093/annonc/mdw040

\section{AUTHOR CONTRIBUTIONS}

$\mathrm{D}-\mathrm{QD}$ and $\mathrm{CZ}$ designed the study; $\mathrm{CZ}$ and Y-ZL performed data processing and experiment analysis; $\mathrm{CZ}$ was a major contributor in writing the manuscript. All authors read and approved the final manuscript.

\section{FUNDING}

This study was supported in part by the National Natural Science Foundation of China (No. 81972322).

\section{ACKNOWLEDGMENTS}

We would like to acknowledge the TCGA, GTEx, HPA, and GEO research consortia for providing open access data for our study.

\section{SUPPLEMENTARY MATERIAL}

The Supplementary Material for this article can be found online at: https://www.frontiersin.org/articles/10.3389/fmolb.2021.645470/ full\#supplementary-material

Supplementary Table S1 | Primers sequences used in present study.

Coffer, P. J., and Burgering, B. M. T. (2004). Forkhead-box transcription factors and their role in the immune system. Nat. Rev. Immunol. 4 (11), 889-899. doi: $10.1038 /$ nri1488

Colaprico, A., Silva, T. C., Olsen, C., Garofano, L., Cava, C., Garolini, D., et al. (2016). TCGAbiolinks: an R/Bioconductor package for integrative analysis of TCGA data. Nucleic Acids Res. 44 (8), e71. doi:10.1093/nar/gkv1507

Dou, C., Jin, X., Sun, L., Zhang, B., Han, M., and Li, T. (2017). FOXF2 deficiency promotes hepatocellular carcinoma metastasis by inducing mesenchymalepithelial transition. Cbm 19 (4), 447-454. doi:10.3233/CBM-170139

Ebrahimi, V., Soleimanian, A., Ebrahimi, T., Azargun, R., Yazdani, P., Eyvazi, S. et al. (2020). Epigenetic modifications in gastric cancer: Focus on DNA methylation. Gene 742, 144577. doi:10.1016/j.gene.2020.144577

Hauptman, N., Jevšinek Skok, D., Spasovska, E., Boštjančič, E., and Glavač, D. (2019). Genes CEP55, FOXD3, FOXF2, GNAO1, GRIA4, and KCNA5 as potential diagnostic biomarkers in colorectal cancer. BMC Med. Genomics 12 (1), 54. doi:10.1186/s12920-019-0501-z

Hirata, H., Ueno, K., Shahryari, V., Deng, G., Tanaka, Y., Tabatabai, Z. L., et al. (2013). MicroRNA-182-5p promotes cell invasion and proliferation by down regulating FOXF2, RECK and MTSS1 genes in human prostate cancer. PLoS One 8 (1), e55502. doi:10.1371/journal.pone.0055502

Huang, K. K., Ramnarayanan, K., Zhu, F., Srivastava, S., Xu, C., Tan, A. L. K., et al. (2018). Genomic and Epigenomic Profiling of High-Risk Intestinal Metaplasia Reveals Molecular Determinants of Progression to Gastric Cancer. Cancer Cell 33 (1), 137-150. e135. doi:10.1016/j.ccell.2017.11.018

Huang, X. x., Zhang, Q., Hu, H., Jin, Y., Zeng, A. l., Xia, Y. b., et al. (2020). A novel circular RNA circFN1 enhances cisplatin resistance in gastric cancer via sponging miR-182-5p. J. Cel Biochem 122, 1009-1020. doi:10.1002/ jcb. 29641

IARC (2012). Personal habits and indoor combustions. Volume 100 E. A review of human carcinogens. IARC Monogr. Eval. Carcinog Risks Hum. 100 (Pt E), $1-538$.

Katoh, M., and Katoh, M. (2004). Human FOX gene family (Review). Int. J. Oncol. 25 (5), 1495-1500. doi:10.3892/ijo.25.5.1495 
Koch, A., Jeschke, J., Van Criekinge, W., van Engeland, M., and De Meyer, T. (2019). MEXPRESS update 2019. Nucleic Acids Res. 47 (W1), W561-W565. doi:10.1093/nar/gkz445

Kong, P.-Z., Li, G.-M., Tian, Y., Song, B., and Shi, R. (2016). Decreased expression of FOXF2 as new predictor of poor prognosis in stage I nonsmall cell lung cancer. Oncotarget 7 (34), 55601-55610. doi:10.18632/ oncotarget.10876

Kundu, S. T., Byers, L. A., Peng, D. H., Roybal, J. D., Diao, L., Wang, J., et al. (2016). The miR-200 family and the miR-183 96 182 cluster target Foxf2 to inhibit invasion and metastasis in lung cancers. Oncogene 35 (2), 173-186. doi:10.1038/ onc.2015.71

Laska, E., Meisner, M., and Wanderling, J. (2012). A maximally selected test of symmetry about zero. Statist. Med. 31 (26), 3178-3191. doi:10.1002/sim.5384

Li, J.-H., Liu, S., Zhou, H., Qu, L.-H., and Yang, J.-H. (2014). starBase v2.0: decoding miRNA-ceRNA, miRNA-ncRNA and protein-RNA interaction networks from large-scale CLIP-Seq data. Nucl. Acids Res. 42, D92-D97. doi:10.1093/nar/gkt1248

Li, L.-C., and Dahiya, R. (2002). MethPrimer: designing primers for methylation PCRs. Bioinformatics 18 (11), 1427-1431. doi:10.1093/bioinformatics/ 18.11.1427

Liang, Y., Zhang, C.-D., Zhang, C., and Dai, D.-Q. (2020). DLX6-AS1/miR-204-5p/ OCT1 positive feedback loop promotes tumor progression and epithelialmesenchymal transition in gastric cancer. Gastric Cancer 23 (2), 212-227. doi:10.1007/s10120-019-01002-1

Lin, J., Shen, J., Yue, H., and Cao, Z. (2019). miRNA-183-5p.1 -p-romotes the -migration and -i-nvasion of -g-astric -c-ancer AGS -c-ells by -t-argeting TPM1. Oncol. Rep. 42 (6), 2371-2381. doi:10.3892/or.2019.7354

Lo, P.-K., Lee, J. S., Liang, X., and Sukumar, S. (2016). The dual role of FOXF2 in regulation of DNA replication and the epithelial-mesenchymal transition in breast cancer progression. Cell Signal. 28 (10), 1502-1519. doi:10.1016/ j.cellsig.2016.06.021

Lu, J.-T., Tan, C.-C., Wu, X.-R., He, R., Zhang, X., Wang, Q.-S., et al. (2020). FOXF2 deficiency accelerates the visceral metastasis of basal-like breast cancer by unrestrictedly increasing TGF- $\beta$ and miR-182-5p. Cell Death Differ 27 (10), 2973-2987. doi:10.1038/s41418-020-0555-7

Pathan, M., Keerthikumar, S., Ang, C.-S., Gangoda, L., Quek, C. Y. J., Williamson, N. A., et al. (2015). FunRich: An open access standalone functional enrichment and interaction network analysis tool. Proteomics 15 (15), 2597-2601. doi:10.1002/pmic.201400515

Plummer, M., Franceschi, S., Vignat, J., Forman, D., and de Martel, C. (2015). Global burden of gastric cancer attributable toHelicobacterpylori. Int. J. Cancer 136 (2), 487-490. doi:10.1002/ijc.28999

Puneet, H. R., Kazmi, S., Tiwari, S., Khanna, A., and Narayan, G. (2018). Epigenetic Mechanisms and Events in Gastric Cancer-Emerging Novel Biomarkers. Pathol. Oncol. Res. 24 (4), 757-770. doi:10.1007/s12253-018-0410-Z

Ritchie, M. E., Phipson, B., Wu, D., Hu, Y., Law, C. W., Shi, W., et al. (2015). limma powers differential expression analyses for RNA-sequencing and microarray studies. Nucleic Acids Res. 43 (7), e47. doi:10.1093/nar/gkv007

Robinson, M. D., and Oshlack, A. (2010). A scaling normalization method for differential expression analysis of RNA-seq data. Genome Biol. 11 (3), R25. doi:10.1186/gb-2010-11-3-r25

Song, J., Liu, Y., Wang, T., Li, B., and Zhang, S. (2020). MiR-17-5p promotes cellular proliferation and invasiveness by targeting RUNX3 in gastric cancer. Biomed. Pharmacother. 128, 110246. doi:10.1016/j.biopha.2020.110246
Sonohara, F., Inokawa, Y., Hayashi, M., Kodera, Y., and Nomoto, S. (2017). Epigenetic modulation associated with carcinogenesis and prognosis of human gastric cancer. Oncol. Lett. 13 (5), 3363-3368. doi:10.3892/ol.2017.5912

Tian, H.-P., Lun, S.-M., Huang, H.-J., He, R., Kong, P.-Z., Wang, Q.-S., et al. (2015). DNA Methylation Affects the SP1-regulated Transcription of FOXF2 in Breast Cancer Cells. J. Biol. Chem. 290 (31), 19173-19183. doi:10.1074/jbc.M114.636126

Tong, Z., Cui, Q., Wang, J., and Zhou, Y. (2019). TransmiR v2.0: an updated transcription factor-microRNA regulation database. Nucleic Acids Res. 47 (D1), D253-D258. doi:10.1093/nar/gky1023

Wang, Q.-S., Kong, P.-Z., Li, X.-Q., Yang, F., and Feng, Y.-M. (2015). FOXF2 deficiency promotes epithelial-mesenchymal transition and metastasis of basallike breast cancer. Breast Cancer Res. 17, 30. doi:10.1186/s13058-015-0531-1

Yuan, C., Zhang, Y., Tu, W., and Guo, Y. (2019). Integrated miRNA profiling and bioinformatics analyses reveal upregulated miRNAs in gastric cancer. Oncol. Lett. 18 (2), 1979-1988. doi:10.3892/ol.2019.10495

Zhang, C., Zhang, C.-D., Liang, Y., Wu, K.-Z., Pei, J.-P., and Dai, D.-Q. (2020). The comprehensive upstream transcription and downstream targeting regulation network of miRNAs reveal potential diagnostic roles in gastric cancer. Life Sci. 253, 117741. doi:10.1016/j.lfs.2020.117741

Zhang, C., Zhao, N., Zhang, X., Xiao, J., Li, J., Lv, D., et al. (2020). SurvivalMeth: a web server to investigate the effect of DNA methylation-related functional elements on prognosis. Brief Bioinform 22, bbaa162. doi:10.1093/bib/bbaal62

Zhang, C., Zou, Y., and Dai, D.-Q. (2019). Downregulation of microRNA-27b-3p via aberrant DNA methylation contributes to malignant behavior of gastric cancer cells by targeting GSPT1. Biomed. Pharmacother. 119, 109417. doi:10.1016/j.biopha.2019.109417

Zhang, J., Liu, H., Hou, L., Wang, G., Zhang, R., Huang, Y., et al. (2017). Circular RNA_LARP4 inhibits cell proliferation and invasion of gastric cancer by sponging miR-424-5p and regulating LATS1 expression. Mol. Cancer 16 (1), 151. doi:10.1186/s12943-017-0719-3

Zhang, Y., Wang, X., Wang, Z., Tang, H., Fan, H., and Guo, Q. (2015). miR-182 promotes cell growth and invasion by targeting forkhead box F2 transcription factor in colorectal cancer. Oncol. Rep. 33 (5), 2592-2598. doi:10.3892/ or.2015.3833

Zuo, Z., Hu, H., Xu, Q., Luo, X., Peng, D., Zhu, K., et al. (2020). BBCancer: an expression atlas of blood-based biomarkers in the early diagnosis of cancers. Nucleic Acids Res. 48 (D1), D789-D796. doi:10.1093/nar/gkz942

Conflict of Interest: The authors declare that the research was conducted in the absence of any commercial or financial relationships that could be construed as a potential conflict of interest.

Publisher's Note: All claims expressed in this article are solely those of the authors and do not necessarily represent those of their affiliated organizations, or those of the publisher, the editors and the reviewers. Any product that may be evaluated in this article, or claim that may be made by its manufacturer, is not guaranteed or endorsed by the publisher.

Copyright (๑) 2021 Zhang, Li and Dai. This is an open-access article distributed under the terms of the Creative Commons Attribution License (CC BY). The use, distribution or reproduction in other forums is permitted, provided the original author(s) and the copyright owner(s) are credited and that the original publication in this journal is cited, in accordance with accepted academic practice. No use, distribution or reproduction is permitted which does not comply with these terms. 